\title{
System Redundancy Optimization with Uncertain Stress-based Component Reliability: Minimization of Regret
}

\author{
Nida Chatwattanasiri \\ National Electronics and Computer Technology Center (NECTEC) \\ Pathum Thani, Thailand \\ David W. Coit ${ }^{*}$ \\ Department of Industrial \& Systems Engineering \\ Rutgers University, Piscataway, New Jersey, USA \\ Naruemon Wattanapongsakorn \\ Department of Computer Engineering \\ King Mongkut's University of Technology Thonburi, Bangkok, Thailand
}

Keywords: system reliability, future usage stress, decision-making with uncertainty, regret

Abstract - System reliability design optimization models have been developed for systems exposed to changing and diverse stress and usage conditions. Uncertainty is addressed through defining a future operating environment where component stresses have shifted or changed for different future usage scenarios. Due to unplanned variations or changing environments and operating stresses, component and system reliability often cannot be predicted or estimated without uncertainty. Component reliability can vary due to a relative increase/decrease of stresses or operating conditions. The uncertain parameters of stresses have been incorporated directly into the new decision-making model. Risk analysis perspectives, including risk-neutral and risk-averse, are considered as system reliability objective functions. A regret function is defined, and minimization of the maximum regret provides an objective function based on random future usage stresses. This is an entirely new formulation of the redundancy allocation problem, but it is a relevant one for some problem domains. The redundancy allocation problem is solved to select the best design solution when there are multiple choices of components and system-level constraints. Nonlinear programming and a neighborhood search heuristic method are recommended to obtain the integer solutions for risk-based formulations.

\subsection{Introduction}

System reliability optimization problems become more realistic when considering uncertainty from associated stresses, operating conditions, etc. for different components within a system. With changing

* Corresponding author, email:coit@ rci.rutgers.edu, telepnone:1-848-445-2033, fax:1-732-445-5467 
loads and stresses in the foreseeable future, a reliability model is useful to predict or determine the impact from these future usage profiles. This new model pertains to applications where it is known that stresses or operating conditions of the system will change, but it is not known the extent of the change, although possible future outcomes can be defined and enumerated.

Consider a new system design where decisions must be made regarding the components to be used, i.e., the number of redundant components and the system architecture. The Redundancy Allocation Problem (RAP) is a well-known problem solved to determine an optimal system configuration. RAP is already a difficult problem; however, now we consider that available component reliability is affected by uncertain future stresses and usage conditions. Design decisions must be made given there are multiple future usage conditions or profiles that can occur.

Aircraft launcher and recovery systems provide some of the motivation to model the anticipated future reliability of components or systems $[1,2,3]$. These systems must operate at a very high level of reliability yet it is anticipated that the airplanes using these systems will be getting heavier due to changing mission types with more required equipment, and also the distribution of airplanes using these systems will be shifting (heavier airplanes will conduct more missions). A particular aircraft launcher or recovery system is exposed to a random pattern of different airplane types, with different characteristics (weights, speeds), creating important and unique reliability issues.

RAP models are presented in this paper where opportunity loss or a regret minimization approach is proposed to directly accommodate uncertainty within the component and system reliability functions. The model formulations considering uncertainty provide additional modeling capabilities and have advantages when compared to traditional reliability models that do not account for risk and uncertainty. The model is realistic and can be applied to various industrial problems, as the uncertainty of system configurations becomes a significant issue in industry.

\subsection{Assumptions}

- Components and systems have two states (fully functional, failed).

- Failure times of individual components are independent. 
- Component failure times follow parametric Weibull distributions.

- Component failure time distributions change in response to different stress levels according to lifetime proportional models (often known as accelerated failure time models).

- Failed components do not inflict any damage on other components, and systems are nonrepairable.

- All redundancy is active redundancy. Components fail at the same rate whether they are a primary or redundant component.

- Operating and usage stress profile will undergo a single shift from the current profile to a different one.

\subsection{Notation}

$$
\begin{aligned}
& R(\mathbf{x} ; t)=\text { system reliability as a function of design vector } \mathbf{x} \text { at time } t \\
& \mathbf{x}=\left(\mathbf{x}_{11}, \mathbf{x}_{12}, \mathbf{x}_{13}, \ldots, \mathbf{x}_{s m_{s}}\right) \\
& x_{i j}=\text { number of identical components for a particular choice } j^{\text {th }} \text { to be used in subsystem } i \\
& s=\text { number of subsystems in a series system } \\
& m_{i}=\text { number of available component selection types or choices for subsystem } i \\
& \mathbf{U}=\text { random future usage profile vector, } \mathbf{U}=\left(U_{1}, U_{2}, \ldots, U_{c}\right), \mathbf{U} \in\left\{\mathbf{u}_{1}, \mathbf{u}_{2}, \ldots, \mathbf{u}_{v}\right\} \\
& U_{k}=\text { stress factor } k \text { (a random variable) } \\
& c=\text { number of different operating usage and stress factors } \\
& \mathbf{u}_{l}=\text { usage profile vector for future usage } l, \mathbf{u}_{l}=\left(u_{1 l}, u_{2 l}, \ldots, u_{c l}\right) \\
& u_{k l}=\text { stress factor } k \text { in future usage } l \\
& p_{l}=\text { probability or likelihood of future usage } l, l=1,2, . ., v \\
& v=\text { number of future usage scenarios } \\
& r_{i j}\left(\mathbf{u}_{l} ; t\right)=\text { reliability for } j^{\text {th }} \text { component choice to be used in subsystem } i \text { in future usage } l \\
& \eta_{i j}\left(\mathbf{u}_{l}\right)=\text { Weibull scale parameter of } j^{\text {th }} \text { component choice in subsystem } i \text { for future usage } l \\
& \eta_{0 i j}=\text { current Weibull scale parameter for } j^{\text {th }} \text { component choice to be used in subsystem } i \\
& \beta_{i j}=\text { Weibull shape parameter of } j^{\text {th }} \text { component choice in subsystem } i \\
& \alpha_{i j k}=\text { sensitivity coefficient of stress factor } k \text { for the } j^{\text {th }} \text { component choice in subsystem } i \\
& W=\text { weight constraint } \\
& C=\text { cost constraint } \\
& w_{i j}=\text { weight of } j^{\text {th }} \text { component choice in subsystem } i \\
& c_{i j}=\operatorname{cost} \text { of } j^{\text {th }} \text { component choice in subsystem } i
\end{aligned}
$$

\subsection{Redundancy Allocation Problem (RAP)}

This research is focused on series-parallel systems with $s$ subsystems connected in series. Within each subsystem $i$, there are potentially redundant components of different choices connected in parallel 
as depicted by the example in Figure 1. All redundancy is active redundancy. The numbers in the figure represent the particular component choice $j$. The number of identical components for a particular choice is $x_{i j}$, and all the components are connected in parallel within each subsystem. In the example in Figure 1 , there are $m_{i}=5$ functionally equivalent component choices for each subsystem, and $n_{i}$ is the sum of all $x_{i j}$ in subsystem $i$. For example, there are two identical components of choice 1 , one component of choice 2 for subsystem 1 and three total components $\left(x_{11}=2, x_{12}=1, x_{13}=x_{14}=x_{15}=0, n_{1}=3\right)$; two identical components of choice 3 for subsystem $2\left(x_{23}=2, x_{21}=x_{22}=x_{24}=x_{25}=0, n_{2}=2\right)$; and for subsystem $3, x_{31}=x_{32}=x_{33}=0, x_{34}=3, x_{35}=1, n_{3}=4$, and so on. Components for a subsystem are selected by solving the RAP subject to system-level cost and weight constraints.

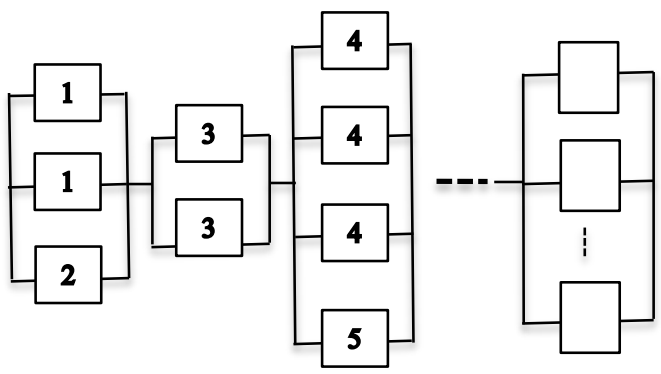

Figure 1. Series-parallel system with multiple choices of components in each subsystem System reliability with deterministic component reliability and active redundancy is $R(\mathbf{x} ; t)=\prod_{i=1}^{s}\left(1-\prod_{j=1}^{m_{i}}\left(1-r_{i j}(t)\right)^{x_{i j}}\right)$ where based on the Weibull distribution, $r_{i j}(t)=\exp \left(-\left(t / \eta_{i j}\right)^{\beta_{i j}}\right)$. The reliability models in this paper are all for applications with active redundancy (or hot standby) and Weibull distributed component failure times (with constant stress levels). The Weibull distribution is a widely applied and flexible distribution, so this is not very restrictive. On the other hand, an important future extension to these models will be to make them more general and to apply to cold-standby redundancy and mixed redundancy types.

RAP considering uncertain conditions was studied by Hada et al $[2,3]$. They evaluated the use of stress covariates for changing stress profiles for aircraft systems. Component-level methods were used to model stress functions for future reliability predictions. A general modeling approach for 
components with changing future stress levels was presented by Johnson et al [1].

To compensate for uncertainty in the RAP, risk minimization can be considered when selecting a system reliability objective function. This stochastic optimization problem can be transformed to an equivalent deterministic problem by defining a future usage stress profile composed of discrete usage or stress scenarios. A risk-neutral approach is to maximize the expected value of the uncertain system reliability. However, if the consequences of low reliability are very dire or undesirable, it may be too risky to use the expected value as an objective function. Even if it is unlikely, the worst or most extreme conditions can occur sometimes, and for some applications, it is important that the system be maximally reliable even then. In these cases, it may be advantageous to use an alternative optimization strategy. The system designer can adopt a minimax regret with robust decision criteria to address uncertainty over possible usage scenarios. In this approach, a regret function is defined and the objective is to minimize the maximum regret given the uncertainty.

For RAP with uncertainty, a decision-maker needs to decide whether they are risk-neutral or riskaverse. The optimal decision is generally different for the two formulations, although for same applications they can be very similar. Given many opportunities, the risk-neutral decision-maker achieves higher system reliability more often. However, they may occasionally achieve unsatisfactorily lower reliability. The risk-averse decision-maker is concerned with the least desirable solutions even if the probability is low. The risk-averse decision-maker may have marginally lower reliability more often, but they will more rarely have very poor reliability.

For decision-making with an uncertain performance criterion, the 'regret' of a decision can be defined as the relative performance loss when compared with the optimal decision which would have been made if perfect knowledge was available. When full knowledge of the underlying model is not available, it is important to consider uncertainty directly in the decision-making process.

\subsection{Background}

Reliability optimization and RAP has been thoroughly and extensively studied. System reliability 
optimization research usually assumes that the components in the system have reliability values that are already known, i.e., deterministic. This assumption, however, is not always appropriate. RAP models have already been formulated and solved many times with known component reliability [4,5]. Ardakan et al [6] studies RAP when both active and standby strategies can be used in a specific system. Discount policies have also been included in RAP problems and two approaches of all unit discount and incremental discount policies were used [7].

The optimal design of a reliable system with the presence of uncertainty has also been investigated in previous research. Coit [8] developed a flexible procedure to approximate confidence intervals for system reliability when there is uncertainty in component reliability information. An optimization model considering risk profiles of system designers and users has also been formulated [9]. This work was further advanced by Coit \& Smith [10] who used a genetic algorithm (GA) to maximize a lower bound for system failure time [11] when there is uncertainty in the Weibull distribution parameters. Coit et al. [12] extended this work by assuming that component and system reliability are random variables. Multiple objective optimization methods were then applied to maximize system reliability and to simultaneously minimize the associated variance. Marseguerra et al. [13] further investigated optimal network design in the presence of component reliability uncertainty by combining Monte Carlo simulation with multiple objective GA.

Recent research pertaining to RAP considering uncertainty was performed by Tekiner-Mogulkoc \& Coit $[14,15]$. Integer programming combined with a search heuristic was used to select the optimal system design with uncertain component reliability estimation, with an objective function to minimize the coefficient of variation (CV). Assuming uncertainty of a failure threshold for the reliability analysis was extended to a degradation model as well [16]. RAP was studied in [17] considering uncertainty by considering cumulative distributions of capacity for a series-parallel system. Taflanidis et al [18] implemented a stochastic subset optimization algorithm.

Bhunia et al [19] presented a nonlinear integer programming problem for maximizing the overall 
system reliability under chance constraints. Several other papers proposed the constrained RAP with interval valued reliability of each component [19-22]. Although several approaches have been proposed for system reliability optimization considering uncertainty, these approaches have not considered uncertain stress directly as it relates to component and system reliability and risk.

The concept of regret has not used often for system reliability optimization, but it is a logical approach when considering uncertainty. Regret analysis has been studied previously for consideration by risk-averse decision-makers. Regret is an emotion often observed in research of decision-making choices. Savage [23] defined the term 'regret' in Savage's minimax regret criterion as the difference between the actual pay-off one received for the decision he made and the optimal pay-off that could have obtained if the best decision was made. Generalization of the regret approach was developed by Loomes et. al [24]. The early regret studies were presented by Kahneman et al. [25] and Bell [26]. The retrospective regret was studied by Gilovich et al. [27] and they proposed a regret model called decision justification theory [25-28]. Making a poor decision under uncertainty can lead to disappointment when alternative choices would have been preferable. Risk analysis and reliability analysis have been discussed previously in the literature $[29,30]$.

For system reliability evaluation, Feizollahi et al [22] proposed the linear-transformed optimization model with constraints. They implemented robust deviation called minimax regret to address uncertainty of component reliability. Feizollahi et al [31] extended the constrained redundancy optimization problem such that component reliabilities belong to a uncertainty set.

In our new model, RAP is extended by relating uncertain stress variables to component reliability with different system perspectives, including regret or opportunity loss analysis. Future usage profiles are enumerated and associated optimization models are formulated and solved. The model presented in this paper is general and can be applied to a wide range of possible system design problems where the component reliability is uncertain or depend on uncertain stress variables. 


\subsection{Uncertain stresses in future usages}

System reliability predictions or estimates can be inaccurate or subject to uncertainty due to unplanned variation or changing operating stresses [32,41]. Future usages of components and systems include changing operating conditions and stresses, and also uncertain environments such as average temperature or usage rates. In general, the stress variables that influence component reliability can be generally classified as (i) environment, (ii) mechanical or electrical stresses, (iii) operating conditions, and (iv) others. The categorization is not rigid, and some stress variables could be categorized in different ways or even included in more than one category. In our model, we collectively refer to them as "stress factors" or "stress variables." Stress is defined generally as any mechanism or influence that affects reliability. Component reliability is defined as a function of these random stress variables.

The future usage profile is composed of a collection of specified stresses in discrete scenarios. Component stress/load parameters are collectively represented as vectors, corresponding to each future usage scenario, which define the stress values in different future usage scenarios. The scenarios are selected to represent the diversity of the possible future usage variables.

Uncertain future usage stress factors are represented in the form of a random vector $\mathbf{U}$. The vector $\mathbf{U}=\left(U_{1}, U_{2}, \ldots, U_{c}\right)$ includes $c$ different operating usage and stress factors and each uncertain usage factor has a different impact on the choice of components. $U_{k}$ is a random variable, which denotes the $k^{\text {th }}$ stress factor, e.g., usage rate, average temperature, average humidity, voltage, etc.

The future usage profile represents a collection of possible future usage conditions that the system may experience with a certain likelihood or probability. These usage scenarios can often be enumerated based on the anticipated missions or environments that the system is anticipated to be operating in or exposed to. Reliability of each component is a function of the random future usage vector $\mathbf{U}$.

The future usage vector $\mathbf{U}$ is random, but in our model, it will be one of a defined set of future usage vectors, $\mathbf{U} \in\left\{\mathbf{u}_{1}, \mathbf{u}_{2}, \ldots, \mathbf{u}_{v}\right\}$. The properties associated with scenario definition are: 
- $\mathbf{u}_{l}$ is a determined vector of future usage in scenario $l, \mathbf{u}_{l}=\left(u_{1 l}, u_{2 l}, \ldots, u_{c l}\right)$

- $u_{k l}$ is a determined variable value or level for the $k^{\text {th }}$ stress factor in scenario $l$

- The current operating condition and stress vector is $\mathbf{u}_{0}$ and assumed to be known.

- The stress variables have all been scaled from 0 to 1 with $\mathbf{u}_{0}=\mathbf{0}$.

In Figure 2, the possible future usage vectors in the future usage profile are defined and each specific future usage scenario is associated with a probability $p_{l}$. For example, for electronic components, average temperature $\left(u_{1 l}\right)$ is a critical contributor to component failure and the risk of component failure increases with increasing average temperatures. For mechanical components, mechanical loading $\left(u_{2 l}\right)$ and stress $\left(u_{3 l}\right)$ are important factors. In practice, a future usage stress could be lower than the current stress level $\left(u_{k l}<0\right)$, but we have assumed that is not the case. The future usage profile with assigned likelihoods is used to model the component and system reliabilities. The failure time of each component is distributed as a Weibull distribution with a scale parameter that depends on future usage scenarios.

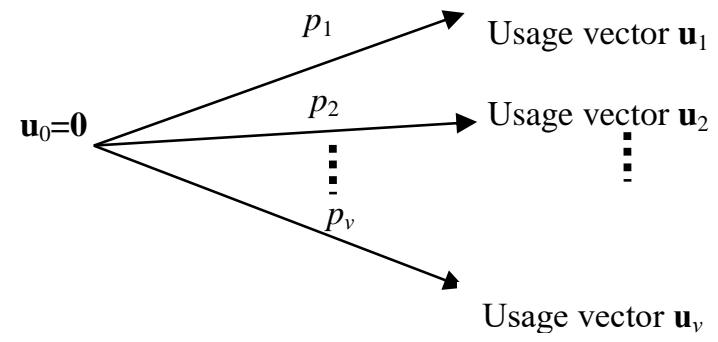

Figure 2. Discrete scenarios in future usage profile

In our formulation, the usage vector $\mathbf{U}$ will change to one of the future usage scenarios vectors $\mathbf{u}_{l}$, $\mathbf{U} \in\left\{\mathbf{u}_{1}, \mathbf{u}_{2}, \ldots, \mathbf{u}_{v}\right\}$, and then will remain there. A related problem would be one where the usage vector changes periodically from one scenario to another at different time intervals. This is a logical extension to the current paper.

Consider the reliability of large-scale IT installations which must have high reliability. Several papers [35-37] reported the failure behavior of a computer drive depends on the system operating conditions. Schroeder et al [36] studied hard drive cluster failure rates affected by environmental factors, such as average temperature and average humidity, data center handling procedures, workloads 
or powered-on hourly patterns, all of which could be considered as stress factors, $U_{k}$.

The discrete future usage scenarios are defined with certain probabilities selected from available information to construct the future usage profile. Each future usage scenario is associated with a probability $p_{l}$ which defines the likelihood of that particular scenario. Expert opinions, historical data or surveys are references that can be used to evaluate the likelihood indexes or probabilities. In practice, these probabilities may not be available, but estimates from the best available knowledge can help to explain how stress contributes to component failure in all probabilistic future scenarios.

There are $v$ possible discrete scenarios in the future usage profile. In practice, the number of discrete scenarios can be infinity. However, the future usage profile embraces most explainable circumstances. $v$ is selected as the minimum number of scenarios to fully characterize the variability in data. Summation over likelihood indexes from all possible scenarios is one, i.e., $\sum_{l=1}^{v} p_{l}=1$.

Scenario definition and selection have been studied extensively within the financial research community and we borrow and exploit their findings. The portfolio selection problem originated from Markowitz [33] takes into account high-order moments in financial engineering. The problem of scenario selection with uncertainties has been used in the portfolio selection problem [34].

\subsection{Reliability models considering uncertainty}

This new RAP model is fundamentally different from other models $[11,12]$ that consider reliability with uncertainty. This new model is evaluated based on the perspective of stress variation as it relates to component and system reliability. In addition, system reliability is expressed directly as a function of uncertain stresses and then integrated into the optimization models. To predict future reliability and estimate the future failure time distribution, the component and system reliabilities incorporate stress factors by utilizing accelerated lifetime concepts or proportional lifetime models, which are analogous to accelerated testing models. 


\subsection{Component reliability model}

Component reliability models have been developed in this paper based on the Weibull distribution with the random stress factors and coefficients given in the probabilistic future usage profile. The Weibull distribution is widely used in component reliability analyses and has repeatedly been demonstrated to be an appropriate model. Nevertheless, other distribution models could also be applied to component reliability once data analyses provides relevant information, and our models can readily be extended to these cases.

A component-specific Weibull distribution is used to model component failure time, and the distribution shifts in response to changing usage stress factors. For different levels of component usage stress, the component failure time distribution changes according to the values of the operating conditions and stresses. Since it is unknown which future usage scenario will occur, the Weibull distributions for component failure time, and the associated parameters, are also uncertain.

Accelerated life models for translating failure time distributions from one stress level to another can generally be classified into (i) proportional hazards models, where hazard rates increase and decrease proportionally according to a function of stresses, or (ii) proportional lifetime models, where the failure time probability density functions compress or expand proportionally based on an acceleration function or factor. In this paper, we adopt a proportional lifetime approach, which means that for the Weibull distribution, the shape parameter is independent of stress, while the scale parameter is a function of stress. Therefore, uncertainty of stress results in changes or shifts in the Weibull distribution scale parameter [1-3], but the shape parameter is constant. An accelerated life model is developed to incorporate uncertain stresses in the reliability functions.

The general equation for the Weibull scale parameter is Eq. 1. For this model, coefficients $\alpha_{i j k}$ indicate component sensitivity to usage and operating stresses and should either have a physical interpretation or based on empirical observations of the relationships between stress and failure time. The Weibull scale parameter is modeled as a log-linear function commonly used for accelerated life 
testing, as a function of stress factors $\mathbf{u}_{l}$ and coefficients $\alpha_{i j k}$.

$$
\eta_{i j}\left(\mathbf{u}_{l}\right)=\eta_{0 i j} \exp \left(-\sum_{k=1}^{c} \alpha_{i j k} u_{l k}\right)
$$

$\eta_{i j}\left(\mathbf{u}_{l}\right)$ is the Weibull scale parameter as a function of the stress vector for component choice $j$ in subsystem $i$ in future usage scenario $l$. The $u_{l k}$ stress factors may require a transformation. As an example, if average temperature is an important stress factor and the Arrhenius relationship was appropriate, then $u_{j k}$ would be defined as the inverse of temperature expressed in ${ }^{\circ} \mathrm{K}$, and the stress sensitivity coefficient would be the activation energy divided by Boltzman's constant.

Component reliability for random $\mathbf{U}$ is given by $r_{i j}(\mathbf{U} ; t)$. Since $\mathbf{U}$ is a random vector, $r_{i j}(\mathbf{U} ; t)$ is also a random variable. However, within a specific scenario $l$, the component reliability is given as $r_{i j}\left(\mathbf{u}_{l} ; t\right)$ which is a deterministic or constant value for specified time $t$ and $\mathbf{u}_{l}$. For life or failure time proportional models, the Weibull shape parameter is constant when stress is varying due to different usage conditions. The component reliability, $r_{i j}\left(\mathbf{u}_{l} ; t\right)$, is given as Eq. 2.

$$
r_{i j}\left(\mathbf{u}_{l} ; t\right)=\exp \left(-\left(t / \eta_{i j}\left(\mathbf{u}_{l}\right)\right)^{\beta_{i j}}\right)
$$

\subsection{Component stress sensitivity}

The sensitivity of component reliability pertains to the change of reliability due to a relative increase/decrease of operating forces or stresses. Stress coefficients are defined as the component stress sensitivity. Sensitivity coefficients for usage stresses, $\alpha_{i j k}$, model the relative effects of different stresses. Coefficients can either be estimated from test data by conducting tests over a range of conditions, based on physics-of-failure models, or obtained from handbooks or published values, e.g., Arrhenius reaction rates. The usage stress functions are applied with these coefficients to determine failure time distribution models considering the effect of different factors/usage conditions. In practice, these coefficients may not be known and dedicated testing is required to quantify the coefficients prior to design optimization. Alternatively, coefficients and parameters can sometimes be assumed based on similar part types or technologies with the same failure modes and mechanisms.

Typical sensitivity coefficients are listed in Table 1 to provide an illustrative example. Component 
reliability is defined and affected by stresses, e.g., average temperature $\left(U_{1}\right)$, usage rate $\left(U_{2}\right)$, and vibration $\left(U_{3}\right)$, respectively and the estimated $\alpha_{i j k}$ coefficients are given in Table 1 for two component choices in each subsystem (one to three). $\alpha_{i j k}$ provides a relative indication of the component sensitivity to changes in stress. The values of the coefficients reflect the extent that the reliabilities of different components respond to the stress factors. For example, the second component choice of subsystem 2 is not affected by the stress factor $2\left(\alpha_{222}=0\right)$. However, component choice 2 available for subsystem 1 is significantly impacted by the stress factor $1\left(\alpha_{121}=1\right)$ as it changes.

Table 1: Sensitivity of stress coefficients

\begin{tabular}{c|c|ccc|c|ccc}
\hline $\begin{array}{c}\text { Subsystem } \\
i\end{array}$ & $\begin{array}{c}\text { Component } \\
\text { Choice 1 }\end{array}$ & \multicolumn{2}{|c|}{ usage and operating stress $(k)$} & $\begin{array}{c}\text { Component } \\
\text { Choice 2 }\end{array}$ & \multicolumn{2}{c|}{ usage and operating stress $(k)$} \\
\hline 1 & $\alpha_{11 k}$ & 0.1 & 0.4 & 0.5 & $\alpha_{12 k}$ & 1 & 0.8 & 1 \\
\hline 2 & $\alpha_{21 k}$ & 1 & 0 & 0.4 & $\alpha_{22 k}$ & 0.2 & 0 & 0.8 \\
\hline 3 & $\alpha_{31 k}$ & 0.4 & 0.8 & 0.2 & $\alpha_{32 k}$ & 0.5 & 1 & 0.2 \\
\hline
\end{tabular}

\subsubsection{Experimental Bearing Data}

Considering a system of bearings as an example, bearing failure is one of the causes of breakdowns in rotating machinery. Degradation of bearings leading to failure is influenced by shaft rotation speed $\left(U_{1}\right)$ and radial load $\left(U_{2}\right)$. Bearing reliability can be expressed as a function of shaft rotation speed and radial load, and where they are subject to change, scenarios are defined with specific numerical values.

The experimental data from "IEEE PHM 2012 prognostic challenge" [42] are used as a case study for this model. Bearing degradation run-to-failure experiments were performed to gather data on ball bearings with various levels of operating conditions. There are two stress variables, rotational speed $\left(U_{1}\right)$ and load $\left(U_{2}\right)$, which are uncertain for future usage conditions.

Experiments were conducted for the PHM challenge, and data were collected from three different stress levels, namely $i$ ) speed $1800 \mathrm{rpm}$ and load $4000 \mathrm{~N}$, ii) speed $1650 \mathrm{rpm}$ and load $4200 \mathrm{~N}$, and iii) speed $1500 \mathrm{rpm}$ and load $5000 \mathrm{~N}$. There were seventeen observed failures of bearings [42] which were used to estimate component reliability distribution parameters. Bearing failure data and scaled stress values were used to fit parameters based on maximum likelihood estimation (MLE) (using Reliasoft ALTA software) as shown in Table 2 for component 1 . Based on this data analysis, load was a very 
significant variable affecting reliability, but rotational speed was not, so $\alpha_{111}=0$. Component 2 represents an alternate component choice with assumed model parameters. Bearing reliability is given by Eq. 2 for any future scenarios with defined stress and $\alpha_{i j k}$ from Table 2 .

Table 2. Distribution parameters and sensitivity coefficients

\begin{tabular}{|c|c|c|c|c|c|c|c|c|}
\hline \multirow{2}{*}{$\begin{array}{c}\text { Choice } \\
j \\
\end{array}$} & \multicolumn{3}{|c|}{$\begin{array}{l}\text { Coefficients of stress } \\
\text { factors }\left(\alpha_{1 j k}\right)\end{array}$} & \multirow{2}{*}{\multicolumn{2}{|c|}{$\begin{array}{c}\text { Weibull shape } \\
\text { parameter } \\
\beta_{1 j} \\
\end{array}$}} & \multirow{3}{*}{$\begin{array}{c}\begin{array}{c}\text { Initial Weibull } \\
\text { scale }\end{array} \\
\eta_{01 j} \\
38140\end{array}$} & \multirow{3}{*}{$\begin{array}{c}\text { cost } \\
c_{1 j} \\
0.05\end{array}$} & \multirow{3}{*}{$\begin{array}{c}\text { weight } \\
w_{1 j} \\
1.2\end{array}$} \\
\hline & & 1 & 2 & & & & & \\
\hline 1 & $\alpha_{11 k}$ & 0 & 1.86 & $\beta_{11}$ & 3.03 & & & \\
\hline 2 & $\alpha_{12 k}$ & 0 & 2.90 & $\beta_{12}$ & 3.03 & 35542 & 0.25 & 0.1 \\
\hline
\end{tabular}

\subsection{System reliability model}

Uncertainty of stress factors is incorporated directly in the system and component reliability function, and thus, the system and component reliability are no longer deterministic. With consideration of uncertain stress factors, the new system reliability function is given in Eq. 3. $\mathbf{U}$ is a random future usage vector, so $r_{i j}(\mathbf{U} ; t)$ and $R(\mathbf{x}, \mathbf{U} ; t)$ are also random.

$$
R(\mathbf{x}, \mathbf{U} ; t)=\prod_{i=1}^{s}\left(1-\prod_{j=1}^{m_{i}}\left(1-r_{i j}(\mathbf{U} ; t)\right)^{x_{i j}}\right)
$$

The decision-maker must decide whether they are risk-averse or risk-neutral. If the decision-maker desires the highest system reliability on average and is less concerned with possible but unlikely low reliability (as long as there is also the corresponding opportunity for very high reliability), then they are risk-neutral decision-makers and can adopt a risk-neutral formulation. The expected value of system reliability is used as the objective function for risk-neutral decision-makers. Often if the implications for low reliability are not severe or only economic, then decision-makers may prefer the risk-neutral model.

Often the decision about risk-neutral vs. risk-averse relates to the implications of poor reliability. If lower than anticipated reliability is extremely undesirable relating to safety or organizational failures, then the decision-maker is likely to be risk-averse. On the other hand, if the implications of low reliability are not critical, a risk-neutral approach may be preferable. 
The new proposed method, based on opportunity loss/regret, can be preferable for risk-averse system designers for decision-making with uncertainty. Regret is interpreted as the difference between the actual system reliability obtained and the value that would have been obtained if a different and preferred course of action had been chosen once the future is observed (which is only known afterwards), or if the particular future usage scenario was known with certainty in advance.

\subsubsection{Expected system reliability}

The expected value of system reliability is derived based on possible future usages and their corresponding probabilities. The expected system reliability is expressed in Eq. 4 as a function of random vector $\mathbf{U}$ and the number of components for each choice.

$$
E_{\mathbf{U}}[R(\mathbf{x}, \mathbf{U} ; t)]=\sum_{l=1}^{v} p_{l} \prod_{i=1}^{s}\left(1-\prod_{j=1}^{m_{i}}\left(1-r_{i j}\left(\mathbf{u}_{l} ; t\right)\right)^{x_{i j}}\right)
$$

Future usage stress vector $\mathbf{u}_{l}$ and sensitivity coefficient of usage and stress factors $\alpha_{i j k}$ are introduced in Eq. 5. Based on Eqs. 1 and 2, and given probabilities for future usage scenarios, the expectation of system reliability as a function of decision variables $x_{i j}$ is given by,

$$
E_{\mathbf{U}}[R(\mathbf{x}, \mathbf{U} ; t)]=\sum_{l=1}^{v} p_{l} \prod_{i=1}^{s}\left(1-\prod_{j=1}^{m_{i}}\left(1-\exp \left(t /\left(\eta_{0 i j} \exp \left(-\sum_{k=1}^{c} \alpha_{i j k} u_{k l}\right)\right)\right)^{\beta_{i j}}\right)^{x_{i j}}\right)
$$

The mean or expected value of system reliability is used as the objective function when a decisionmaker desires risk-neutral evaluation. The number of components of each choice in the parallel subsystems can be determined by maximizing the expected system reliability.

\subsubsection{Regret of system reliability}

The regret analysis approach for optimizing system reliability is for risk-averse decision-makers. The possible future usage profile includes discrete scenarios, and the objective is to find a solution that performs reasonably well for all scenarios, i.e., "best worst-case" performance to hedge against the possible risk under other scenarios [29-31]. The regret approach is proposed for the risk-averse decision-makers to select the best possible solution that minimizes the maximum potential loss in case a poor decision yields an undesirable large risk. 
Given $v$ future usage scenarios in the future usage profile, $\mathbf{x}_{l} *$ is the optimal solution in each individual scenario $l(l=1,2, \ldots, v)$, if it is known with certainty that particular scenario would occur. Therefore, there are $v$ different $\mathbf{x}_{l}{ }^{*}$ solutions, i.e., one for each scenario. These are determined beforehand by solving $v$ different nonlinear optimization problems. Each optimal solution consists of different component selections that maximize system reliability $\max \left(R\left(\mathbf{x}, \mathbf{u}_{l} ; t\right)\right)$ for that scenario. Regret or loss is denoted by $\Delta_{l}$ as a function of configuration $\mathbf{x}$ in future usage scenario $l$. If $\mathbf{x}$ is chosen instead of $\mathbf{x}_{l}^{*}$ as the solution for scenario $l$, the pairwise regret is defined as

$$
\begin{gathered}
\Delta_{l}=\left|R\left(\mathbf{x}_{l}^{*}, \mathbf{u}_{l} ; t\right)-R\left(\mathbf{x}, \mathbf{u}_{l} ; t\right)\right| \\
\Delta_{l}=R\left(\mathbf{x}_{l}^{*}, \mathbf{u}_{l} ; t\right)-\prod_{i=1}^{s}\left(1-\prod_{j=1}^{m_{i}}\left(1-\exp \left(t /\left(\eta_{0 i j} \exp \left(-\sum_{k=1}^{c} \alpha_{i j k} u_{k l}\right)\right)\right)^{\beta_{i j}}\right)^{x_{i j}}\right) \\
\text { where } \quad R\left(\mathbf{x}_{l}^{*}, \mathbf{u}_{l} ; t\right)=\max _{\mathbf{x}}\left\{R\left(\mathbf{x}, \mathbf{u}_{l} ; t\right)\right\} ; l=\{1,2, \ldots, v\}
\end{gathered}
$$

In contrast to consideration of interval component reliability, our component reliability is evaluated deterministically for each discrete scenario in the future usage profile. For the minimax regret approach $[31,39,40]$, we define a set of scenarios and set of solutions in each scenario of the future usage profile.

\subsection{Optimization models}

The system reliability optimization model has been formulated and solved to minimize maximum regret. The regret or opportunity loss of system reliability is used as the objective function and the expected system reliability is also determined for all problems. The problem can be solved as a nonlinear integer problem either by using a global nonlinear solver designed specifically for these difficult problems or by new heuristic method proposed in this paper. When a global nonlinear integer solver is available, it is the preferred approach to solve the problem. However, when an appropriate solver is not available or integer variable restrictions have been surpassed, then the NS heuristic is proposed as an alternative.

For series-parallel systems, RAP is formulated with decision variables $x_{i j}$. We initially introduce a simple risk-neutral objective to maximize expected system reliability by determining the component 
choices, and the number of components of each choice in each subsystem. As shown in Eq. 8, the expected value of system reliability is maximized considering cost and weight constraints.

$$
\begin{aligned}
& \left.\max \quad E_{\mathbf{U}}[R(\mathbf{x}, \mathbf{U} ; t)]=\sum_{l=1}^{v} p_{l} \prod_{i=1}^{s}\left(1-\prod_{j=1}^{m_{i}} 1-\exp \left(-\left(t / \eta_{i j}\left(\mathbf{u}_{l}\right)\right)^{\beta_{i j}}\right)\right)^{x_{i j}}\right) \\
& \text { s.t. } \sum_{i=1}^{s} \sum_{j=1}^{m_{i}} c_{i j} x_{i j} \leq C, \sum_{i=1}^{s} \sum_{j=1}^{m_{i}} w_{i j} x_{i j} \leq W, x_{i j} \in\{0,1,2, \ldots\}, i=1,2, \ldots, s, j=1,2, \ldots, m_{i}
\end{aligned}
$$

In this optimization problem, each possible future usage $l$ with probability $p_{l}$ for all $l$ is used to compute the expected value of the system reliability. The decision variables $x_{i j}$ are selected for each component choice $j$ at subsystem $i$ to maximize the objective function with cost and weight constraints. For these examples, component and system weight refers the physical weight or mass (measured in $\mathrm{kg}$ or lbs).

In this optimization formulation, the system cost is represented as a summation of the component costs. While this constraint or very similar ones have been used often before $[4,5,8]$, is only a gross approximation of the true costs of developing and operating a system. A realistic and accurate cost function would require many additional factors and considerations including the consumed energy and materials used during operation and many other factors. For some examples of more detailed and rigorous cost modeling, refer to Levitin et al [43-46] for cost models of standby systems.

\subsection{System reliability optimization: Minimizing maximum regret}

Regret analysis is the approach used for RAP with uncertainty and risk-aversion. When the decision-makers are uncertain about the consequences of system reliability under different future usage scenarios and a poor decision can lead to severe risk, the regret formulation is a viable option [28,29]. Solutions that have good performance, even good worst-case performance, for all scenarios are called robust solutions [38].

The objective function is to minimize the maximum pairwise regret or opportunity loss, considering system cost and weight constraints, and given as Eq. 9. The optimal solutions for each specific future usage scenarios are pre-determined and then applied in the model. 


$$
\min \max \text { regret }=\min _{\mathbf{x}} \max _{l} \Delta_{l}=\min _{\mathbf{x}} \max _{l}\left|R\left(\mathbf{x}_{l}^{*}, \mathbf{u}_{l} ; t\right)-R\left(\mathbf{x}, \mathbf{u}_{l} ; t\right)\right|
$$

It is difficult to solve the problem directly, so it is transformed into an equivalent problem, as shown in Eq. 10.

$$
\begin{array}{ll} 
& \min _{\mathbf{x}, z} z \\
\text { s.t. } & R\left(\mathbf{x}_{l}^{*}, \mathbf{u}_{l} ; t\right)-R\left(\mathbf{x}, \mathbf{u}_{l} ; t\right) \leq z ; \forall l \\
& \sum_{i=1}^{s} \sum_{j=1}^{m_{i}} c_{i j} x_{i j} \leq C, \quad \sum_{i=1}^{s} \sum_{j=1}^{m_{i}} w_{i j} x_{i j} \leq W \\
& x_{i j} \in\{0,1,2,3, \ldots\}, i=1,2, \ldots, s, j=1,2, \ldots m_{i}
\end{array}
$$

The regret model requires the solution of $v+1$ different optimization models whereas the expected system reliability model requires the solution of only a single problem. As a result, the expected system reliability model is inherently computational more efficient.

These optimization models have complex nonlinear objective and constraint functions and integer decision variables. These integer nonlinear programming models are very difficult to solve, and heuristics or meta-heuristics are often used for this class of problems. If a global nonlinear integer solver is available to solve the problems to optimality (e.g, LINGO Global Solver), it is the recommended approach to solve the problem, although they potentially require additional software add-ins and have restrictions on the number of allowable integer variables. Alternatively, we have developed a heuristic specifically for this problem that can be more readily applied by commonly available software (e.g., MATLAB optimization toolbox). It is called the NS heuristic and described in the following section.

\subsection{Neighborhood Search (NS) heuristic algorithm}

Efficient heuristic methods can be used as an alternative option to solve these complex large-scale problems and to obtain solutions. A generally effective heuristic is developed to solve this problem based on nonlinear programming and neighborhood search.

The heuristic approach developed for this paper is to initially relax integer constraints and to solve a continuous nonlinear optimization problem. Then a thorough neighborhood search (NS) is applied to 
determine recommended integer solutions that are in close proximity to the continuous solution. The NS heuristic is required for the risk-neutral problem (case 1), for $v$ different initial problems in the regret analysis for each scenario (case 2), and for the minimax problem (case 2). The regret analysis approach requires the solution of $v+1$ different problems, and it is less efficient. To search for the optimal integer number of components of each choice in each subsystem, the heuristic approach selects the best feasible integer solution in the neighborhood [32, 42].

The NS heuristic proceeds as follows:

Step 1: The integer problem is transformed by relaxing integer constraints. The relaxed continuous nonlinear problem is solved and solutions found. Nonlinear optimization using the pattern search solver (e.g., from MATLAB optimization toolbox) is used to find the continuous solutions $\mathbf{x}_{\text {con }}$.

Step 2: Different integer system solutions are enumerated considering all combinations of possible component integer solutions, which are constructed considering the integer solutions immediately higher and lower than the optimal continuous solution. $\mathbf{x}_{c o n}$ represents the relaxed continuous solutions of the optimization model and $\mathbf{x}_{\text {in }}$ represents the integer solutions of the optimization model by creating bounds that are integers immediately lower and higher than the continuous solutions.

$$
\mathbf{x}_{\text {in }} \leftarrow \text { set of }\left\{\left\lfloor\mathbf{x}_{c o n}\right\rfloor, \ldots,\left\lceil\mathbf{x}_{\text {con }}\right\rceil\right\} \text { there are } 2^{s} \text { number of vectors in the set }
$$

For in $=1,2, \ldots, 2^{s}$, compute objective function and evaluate feasibility in Step 3.

For the set of solutions that do not violate a constraint, proceed to Step 4.

Step 3: Check if constraints are violated.

$$
\mathbf{1} \leq \mathbf{x}_{\text {in }} \leq \mathbf{x}_{\max }, \mathbf{c} \mathbf{x}_{\text {in }} \leq C, \mathbf{w} \mathbf{x}_{\text {in }} \leq W
$$

When iterating solutions that violate constraints, the algorithm ignores those infeasible solutions and returns to Step 2 iterations. When feasible, compare objective functions to best found, and retain the best solution and objective function.

$$
\begin{aligned}
& R \leftarrow \max \left\{E\left[R\left(\mathbf{x}_{i n}, \mathbf{U} ; t\right)\right]\right\}, \text { for case } 1 \\
& R^{l} \leftarrow \max \left\{R\left(\mathbf{x}_{i n}, \mathbf{u}_{l} ; t\right)\right\}, \text { for all } l, \text { for case } 2
\end{aligned}
$$

Step 4: The final best feasible solution is the recommended solution as vector $\mathbf{x}^{*}$ Step 5: Calculate system reliability

$$
R_{s} \leftarrow E\left[R\left(\mathbf{x}^{*}, \mathbf{U} ; t\right)\right] \text {, for case } 1
$$




$$
R_{s}^{l} \leftarrow R\left(\mathbf{x}^{*}, \mathbf{u}_{l} ; t\right) \text {, for all } l, \text { for case } 2
$$

For case 2, the regret modeling approach, the system reliability in Step 5 is used for the pairwise of regret calculation of each scenario $l$.

\subsubsection{Procedure of pairwise regrets and maximum regret}

The procedure for minimization of maximum regret analysis (case 2) approach is as follows:

Step 1: The NS heuristic from Section 5.2 is used to initially determine integer solutions for each of the $l$ scenarios.

Step 2: The integer solutions and reliability from solutions in Step 1 are used in Eq. 10.

Step 3: By comparing the pairwise regrets and using the model derived in Eq. 10, the continuous solution that minimizes the maximum regret can be found using the pattern search optimizer (in MATLAB toolbox).

Step 4: The neighborhood of the optimal continuous solutions, as defined in Step 2 of Section 5.2, is enumerated to find the best feasible integer solution in the neighborhood.

\subsection{Examples}

Several examples were solved to demonstrate and evaluate the model. The NS heuristic was used to solve the problems, but later compared to other optimization approaches to the problem. The first example addresses design of a system of bearings, while the second and third examples are hypothetical examples. In the second example, the three stress variables all decrease as the future random scenarios become less stressful, while the third example has more randomly distributed stress variable values creating a more difficult problem and more interesting trade-offs.

\subsection{Example 1: Bearing system design}

The first example continues the bearing descriptions from Section 4.2.1. The system objective is to design a manufacturing system with redundancy added to increase the reliability of a single parallel structure $(s=1)$ for a critical operating duration of $t=15,000$. The experimental data from degradation run-to-failure experiments [42] were used to quantify the component reliability model (which was presented in Section 4.2.1). There are system level constraints of $C=1.0$ and $W=2.0$.

The future random stress variables are rotational speed and load, i.e., $\mathbf{U}=\left(U_{1}, U_{2}\right)$. A future usage 
profile is defined by three future usage scenarios, $\mathbf{U} \in\left\{\mathbf{u}_{1}, \mathbf{u}_{2}, \mathbf{u}_{3}\right\}$, which represent three possible operating condition settings with probabilities $p_{l}=(1 / 3,1 / 3,1 / 3)$ as shown in Table 3 . The stress vectors for the three scenarios $\mathbf{u}_{1}, \mathbf{u}_{2}$ and $\mathbf{u}_{3}$ are scaled between 0 to 1 .

Table 3. Future usage profile of bearing testing

\begin{tabular}{c|c|cc}
\hline \multicolumn{2}{c|}{ Example 1 } & \multicolumn{2}{c}{$u_{k l}$} \\
\hline Probability at future $l$ & $u_{1 l}$ & $u_{2 l}$ \\
\hline$p_{1}$ & $1 / 3$ & 1 & $1 / 3$ \\
$p_{2}$ & $1 / 3$ & $5 / 8$ & $7 / 15$ \\
$p_{3}$ & $1 / 3$ & $1 / 4$ & $3 / 4$
\end{tabular}

From an optimization perspective, this is an easy problem to solve with only two decision variables. In fact, the initial continuous solutions for each of the $l$ scenarios are integers, and are the same solution for all three scenarios $\left(x_{11}=8, x_{12}=0\right)$. Therefore, the maximum regret is zero, and this is the best solution from both risk-averse and risk neutral perspectives. Larger problems are studied in the following sections.

\subsection{Additional examples (Examples 2 and 3)}

The second and third problems are much larger with 14 subsystems $(s=14)$ and between one and four component choices for each subsystem. Ten scenarios with associated stress factors and probabilities are given as the future usage profile for both problems, and presented as Table 4. The problems are similar but with one important difference. For Example 2, the stress variable values are all decreasing with the scenario number for all three stress variables. For Example 3, the third stress variable value has been randomized creating a more interesting problem with more complex trade-offs. For the third example, the relative difference between component choice reliability can vary depending on the stress sensitivity coefficients $\left(\alpha_{i j k}\right)$.

The system reliability optimization is conducted for a critical mission time of $t=100$. Tables 5 to 7 present the component data, which are modified versions of the parameters from Tekiner \& Coit [15]. Table 5 presents component cost, weight and baseline Weibull scale parameter. $\alpha_{i j k}$ coefficients relate to usage stresses, and are given in Table 6. A higher number indicates that the component reliability is 
more sensitive to that stress. In practice, the $\alpha_{i j k}$ coefficients should be determined based on conducting experiments, coefficients from physics-of-failure models or other published data, e.g., Arrhenius reaction rates. For all components considered in these examples and all scenarios, the Weibull shape parameter is given in Table 7 and the scale parameter is given as $\eta_{i j}\left(\mathbf{u}_{l}\right)=\eta_{0 i j} \exp \left(-\left(\alpha_{i j 1} u_{1 l}+\alpha_{i j 2} u_{2 l}+\alpha_{i j 3} u_{3 l}\right)\right.$

Table 4: Future usage profile (example 2 and 3)

\begin{tabular}{c|c|ccc|ccc}
\hline \multicolumn{2}{c|}{ Example 1 } & \multicolumn{3}{c|}{$u_{k l}$ (example 2) } & \multicolumn{3}{c}{$u_{k l}$ (example 3) } \\
\hline \multicolumn{2}{c|}{ Probability at future $l$} & $u_{1 l}$ & $u_{2 l}$ & $u_{3 l}$ & $u_{1 l}$ & $u_{2 l}$ & $u_{3 l}$ \\
\hline$p_{1}$ & 0.04 & 0.95 & 1 & 0.90 & 0.95 & 1 & 0.20 \\
$p_{2}$ & 0.08 & 0.80 & 0.85 & 0.80 & 0.80 & 0.85 & 0.10 \\
$p_{3}$ & 0.08 & 0.75 & 0.78 & 0.75 & 0.75 & 0.78 & 0.10 \\
$p_{4}$ & 0.12 & 0.60 & 0.70 & 0.65 & 0.60 & 0.70 & 0.80 \\
$p_{5}$ & 0.16 & 0.55 & 0.55 & 0.60 & 0.55 & 0.55 & 0.30 \\
$p_{6}$ & 0.15 & 0.42 & 0.42 & 0.49 & 0.42 & 0.42 & 0.20 \\
$p_{7}$ & 0.13 & 0.36 & 0.38 & 0.40 & 0.36 & 0.38 & 0.50 \\
$p_{8}$ & 0.10 & 0.28 & 0.25 & 0.30 & 0.28 & 0.25 & 0.50 \\
$p_{9}$ & 0.09 & 0.19 & 0.18 & 0.17 & 0.19 & 0.18 & 0.70 \\
$p_{10}$ & 0.05 & 0.10 & 0.05 & 0.10 & 0.1 & 0.05 & 0.90 \\
\hline
\end{tabular}

The regret optimization model is solved for both problems. Probabilities of future usage scenarios are not necessary to minimize the worst-case regret, but they are needed to maximize expected system reliability. The scenario probabilities and stress values for these two examples are given in Table 4. The NS heuristic is used to obtain the integer solutions.

Table 5: Example parameters for available choices of components in each subsystem

\begin{tabular}{c|c|c|c|c|c|c|c|c|c|c|c|c|c}
\hline$i$ & choices $\left(m_{i}\right)$ & $\eta_{0 i 1}$ & $c_{i 1}$ & $w_{i 1}$ & $\eta_{0 i 2}$ & $c_{i 2}$ & $w_{i 2}$ & $\eta_{0 i 3}$ & $c_{i 3}$ & $w_{i 3}$ & $\eta_{0 i 4}$ & $c_{i 4}$ & $w_{i 4}$ \\
\hline 1 & 3 & 448.3 & 1 & 3 & 574.8 & 1 & 4 & 1060.3 & 2 & 2 & 724.4 & 2 & 5 \\
2 & 1 & 441.5 & 2 & 8 & 304.4 & 1 & 10 & 574.8 & 1 & 9 & & & \\
3 & 4 & 248.1 & 2 & 7 & 308.1 & 3 & 5 & 372.2 & 1 & 6 & 346.3 & 4 & 4 \\
4 & 3 & 536.7 & 3 & 5 & 268.0 & 4 & 6 & 615.3 & 5 & 4 & & & \\
5 & 2 & 1616.2 & 2 & 4 & 285.6 & 2 & 3 & 1949.6 & 3 & 5 & & & \\
6 & 2 & 2147.2 & 3 & 5 & 4949.8 & 3 & 4 & 3283.1 & 2 & 5 & 2449.7 & 2 & 4 \\
7 & 1 & 325.6 & 4 & 7 & 346.3 & 4 & 8 & 1616.2 & 5 & 9 & & & \\
8 & 1 & 186.4 & 3 & 4 & 308.1 & 5 & 7 & 325.6 & 6 & 6 & & & \\
9 & 3 & 3283.1 & 2 & 8 & 2147.2 & 3 & 9 & 843.5 & 4 & 7 & 482.6 & 3 & 8 \\
10 & 2 & 306.5 & 4 & 6 & 615.3 & 4 & 5 & 949.1 & 5 & 6 & & & \\
11 & 1 & 1616.2 & 3 & 5 & 724.4 & 4 & 6 & 843.5 & 5 & 6 & & & \\
12 & 1 & 262.1 & 2 & 4 & 224.5 & 3 & 5 & 248.1 & 4 & 6 & 448.3 & 5 & 7 \\
13 & 2 & 1348.1 & 2 & 5 & 997.5 & 3 & 5 & 3283.1 & 2 & 6 & & & \\
14 & 3 & 448.3 & 4 & 6 & 1199.3 & 4 & 7 & 1949.6 & 5 & 6 & 997.5 & 6 & 9 \\
\hline
\end{tabular}

NOTE: Italicized numbers are not used in Examples 2 and 3, but were used in the experimental comparisons in Section 6.3 
Table 6: Usage and operating stress coefficients for available components in each subsystem

\begin{tabular}{c|c|ccc|c|ccc}
\hline & \multicolumn{2}{|c|}{ Choice1 } & \multicolumn{2}{|c|}{ usage and operating stress $(k)$} & \multicolumn{2}{c|}{ Choice 2 } & \multicolumn{3}{c}{ usage and operating stress $(k)$} \\
\hline$i$ & $\alpha_{i j k}$ & 1 & 2 & 3 & $\alpha_{i j k}$ & 1 & 2 & 3 \\
\hline $1-4$ & $\alpha_{i 1 k}$ & 0.6 & .45 & 0.2 & $\alpha_{i 2 k}$ & 0.5 & 0.35 & 0.4 \\
$5-8$ & $\alpha_{i 1 k}$ & 0.35 & 0.4 & 0.2 & $\alpha_{i 2 k}$ & 0.3 & 0.55 & 0.8 \\
$9-11$ & $\alpha_{i 1 k}$ & 0.7 & 0.5 & 0.6 & $\alpha_{i 2 k}$ & 0.5 & 0.35 & 0.4 \\
$12-14$ & $\alpha_{i 1 k}$ & 0.4 & 0.1 & 0.4 & $\alpha_{i 2 k}$ & 0.6 & 0.75 & 0.4 \\
\hline & Choice 3 & \multicolumn{2}{|c|}{ usage and operating stress $(k)$} & Choice 4 & usage and operating stress $(k)$ \\
\hline$i$ & $\alpha_{i j k}$ & 1 & 2 & 3 & $\alpha_{i j k}$ & 1 & 2 & 3 \\
\hline $1-4$ & $\alpha_{i 3 k}$ & 0.7 & 0.2 & 0.6 & $\alpha_{i 4 k}$ & 0.4 & 0.8 & 0.3 \\
$5-8$ & $\alpha_{i 3 k}$ & 0.6 & 0.2 & 0.25 & $\alpha_{i 4 k}$ & 0.25 & 0.45 & 0.65 \\
$9-11$ & $\alpha_{i 3 k}$ & 0.7 & 0.2 & 0.6 & $\alpha_{i 4 k}$ & 0.6 & 0.4 & 0.45 \\
$12-14$ & $\alpha_{i 3 k}$ & 0.4 & 0.5 & 0.35 & $\alpha_{i 4 k}$ & 0.6 & 0.5 & 0.3 \\
\hline
\end{tabular}

NOTE: Italicized numbers are not used in Examples 2 and 3, but were used in the experimental comparisons in Section 6.3

\subsubsection{Example 2: Stress factors in decreasing direction}

For this example, the stress factors in the future usage profile are all decreasing for future usage scenarios 1 to 10 as shown in Table 4. The current operating stress vector is given as $\mathbf{u}_{0}=\mathbf{0}$. In future usages, the system or components can experience different usage scenarios with probabilities given in Table 4. For instance, future usage scenario 1 occurs with probability 0.04 and with operating stress vector $\mathbf{u}_{1}=(0.95,1,0.9)$.

Table 7: Weibull shape parameters of available choices of components in each subsystem

\begin{tabular}{c|cccc}
\hline$i$ & Choice 1 & Choice 2 & Choice 3 & Choice 4 \\
\hline subsystem & $\beta_{1}$ & $\beta_{2}$ & $\beta_{3}$ & $\beta_{4}$ \\
\hline 1 & 1.5 & 1.5 & 2 & 1.5 \\
2 & 2 & 2.5 & 1.5 & \\
3 & 1.5 & 2 & 1.5 & 2 \\
4 & 1.5 & 2 & 2 & \\
5 & 2 & 2.5 & 2 & \\
6 & 1.5 & 1.5 & 2 & 1 \\
7 & 1.5 & 2 & 1.5 & \\
8 & 1.5 & 2 & 2 & \\
9 & 1.5 & 1.5 & 1.5 & 1.5 \\
10 & 1.5 & 1.5 & 1 & \\
11 & 2 & 1.5 & 1.5 & \\
12 & 1.5 & 2 & 2 & 1.5 \\
13 & 1.5 & 2 & 2.5 & \\
14 & 1.5 & 1.5 & 1.5 & 2 \\
\hline
\end{tabular}

NOTE: Italicized numbers are not used in Examples 2 and 3, but were used in the experimental comparisons in Section 6.3

To determine regret, it is necessary to initially maximize system reliability for each scenario 
individually and those results are in Table 8. For Example 2, the problem was solved and the minimax opportunity loss or regret obtained is 0.0274 , and the solution $\mathbf{x}^{*}$ is shown in Table 9 . The problem was also solved to maximize expected system reliability, and the results are presented in Table 10 . The solutions to minimize maximum regret and to maximize expected system reliability are similar, but there are distinct differences. Those decision variables that are different are indicated in bold in the tables.

Table 8: Optimal system reliabilities for future usage scenarios (Example 2)

\begin{tabular}{l|cccccccccc}
\hline \multicolumn{1}{c|}{ Future usage $l$} & 1 & 2 & 3 & 4 & 5 & 6 & 7 & 8 & 9 & 10 \\
\hline reliability (continuous solution) & .4695 & .6920 & .7667 & .8715 & .9217 & .9630 & .9745 & .9866 & .9925 & .9960 \\
\hline reliability (integer solution) & .4120 & .6296 & .7023 & .8122 & .8678 & .9213 & .9385 & .9589 & .9711 & .9796 \\
\hline
\end{tabular}

\subsubsection{Example 3: Stress factors are randomized}

In Example 3, the stress factors $u_{3 l}$ are randomly generated as shown in Table 4 to observe how the proposed model performs for a more interesting example. Since the sensitivity coefficients are also not in decreasing order, the selection of components requires a further compromise and more interesting trade-offs.

Table 9: Selections of components in minimax regret approach (Example 2)

\begin{tabular}{|c|c|c|c|c|c|}
\hline \multirow{2}{*}{$\frac{s=14}{x_{i j}}$} & \multicolumn{5}{|c|}{ Regret analysis: max regret $=0.0274$} \\
\hline & $\#$ & $x_{i j}$ & $\#$ & $x_{i j}$ & $\#$ \\
\hline$x_{11}$ & 0 & $x_{43}$ & 3 & $x_{101}$ & 0 \\
\hline$x_{12}$ & 0 & $x_{51}$ & 1 & $x_{102}$ & 3 \\
\hline$x_{13}$ & 3 & $x_{52}$ & 0 & $x_{111}$ & 2 \\
\hline$x_{21}$ & 3 & $x_{61}$ & 0 & $x_{121}$ & 5 \\
\hline$x_{31}$ & 0 & $x_{62}$ & 2 & $x_{131}$ & 2 \\
\hline$x_{32}$ & 4 & $x_{71}$ & 3 & $x_{132}$ & 0 \\
\hline$x_{33}$ & 1 & $x_{81}$ & 6 & $x_{141}$ & 0 \\
\hline$x_{34}$ & $\mathbf{0}$ & $x_{91}$ & 1 & $x_{142}$ & 0 \\
\hline$x_{41}$ & 0 & $x_{92}$ & 0 & $x_{143}$ & 2 \\
\hline$x_{42}$ & 0 & $x_{93}$ & 0 & $\mathbf{c x}=12$ & \\
\hline
\end{tabular}


Table 10: Selections of components for expected system reliability (Example 2)

\begin{tabular}{cc|cc|cc}
\hline$s=14$ & \multicolumn{5}{c}{ Expected System Reliability $=0.8652$} \\
\hline$x_{i j}$ & $\#$ & $x_{i j}$ & $\#$ & $x_{i j}$ & 0 \\
\hline$x_{11}$ & 0 & $x_{43}$ & 3 & $x_{101}$ & 3 \\
$x_{12}$ & 0 & $x_{51}$ & 1 & $x_{102}$ & 2 \\
$x_{13}$ & $\mathbf{2}$ & $x_{52}$ & 0 & $x_{111}$ & 5 \\
$x_{21}$ & 3 & $x_{61}$ & 0 & $x_{121}$ & 2 \\
$x_{31}$ & 0 & $x_{62}$ & $\mathbf{1}$ & $x_{131}$ & 0 \\
$x_{32}$ & $\mathbf{1}$ & $x_{71}$ & $\mathbf{4}$ & $x_{132}$ & 0 \\
$x_{33}$ & $\mathbf{0}$ & $x_{81}$ & $\mathbf{7}$ & $x_{141}$ & 0 \\
$x_{34}$ & $\mathbf{4}$ & $x_{91}$ & 1 & $x_{142}$ & $x_{143}$ \\
$x_{41}$ & 0 & $x_{92}$ & 0 & $\mathbf{c x}=130, \mathbf{w x}=200$ \\
$x_{42}$ & 0 & $x_{93}$ & 0 & 11 & 2 \\
\hline
\end{tabular}

The problem was solved to minimize maximum regret. Table 11 indicates system reliabilities obtained for each possible future usage scenarios. The maximum opportunity loss or regret is found to be 0.0257 , and the compromise solution decision variables $\mathbf{x}^{*}$ are shown in Table 12. Example 3 was also solved to maximize expected system reliability and those results are indicated in Table 13 . The bolded numbers in Tables 12 and 13 indicate where the solutions are different.

Table 11: Optimal system reliabilities for future usage scenarios (Example 3)

\begin{tabular}{l|cccccccccc}
\hline \multicolumn{1}{c|}{ Future usage $l$} & 1 & 2 & 3 & 4 & 5 & 6 & 7 & 8 & 9 & 10 \\
\hline reliability (continuous solution) & .7652 & .8824 & .9065 & .8409 & .9508 & .9768 & .9700 & .9813 & .9818 & .9844 \\
\hline reliability (integer solution) & .7011 & .8295 & .8584 & .7831 & .9083 & .9429 & .9316 & .9481 & .9475 & .9497 \\
\hline
\end{tabular}

Table 12: Selections of components in minimax regret approach (Example 3)

\begin{tabular}{cc|cc|cc}
\hline$s=14$ & \multicolumn{5}{c}{ Regret analysis: max regret $=0.0257$} \\
\hline$x_{i j}$ & $\#$ & $x_{i j}$ & $\#$ & $x_{i j}$ & $\#$ \\
\hline$x_{11}$ & 0 & $x_{43}$ & 3 & $x_{101}$ & 0 \\
$x_{12}$ & 0 & $x_{51}$ & $\mathbf{1}$ & $x_{102}$ & 3 \\
$x_{13}$ & $\mathbf{3}$ & $x_{52}$ & 0 & $x_{111}$ & 2 \\
$x_{21}$ & 3 & $x_{61}$ & 0 & $x_{121}$ & 5 \\
$x_{31}$ & 0 & $x_{62}$ & 1 & $x_{131}$ & 1 \\
$x_{32}$ & $\mathbf{2}$ & $x_{71}$ & $\mathbf{3}$ & $x_{132}$ & 1 \\
$x_{33}$ & $\mathbf{2}$ & $x_{81}$ & 7 & $x_{141}$ & 0 \\
$x_{34}$ & $\mathbf{1}$ & $x_{91}$ & 1 & $x_{142}$ & 0 \\
$x_{41}$ & 0 & $x_{92}$ & 0 & $x_{143}$ & 2 \\
$x_{42}$ & 0 & $x_{93}$ & 0 & $\mathbf{c x}=122, \mathbf{w x}=200$ \\
\hline
\end{tabular}


Table 13: Selections of components for expected system reliability (Example 3)

\begin{tabular}{cl|ll|ll}
\hline$s=14$ & \multicolumn{5}{c}{ Expected System Reliability $=0.9053$} \\
\hline$x_{i j}$ & $\#$ & $x_{i j}$ & $\#$ & $x_{i j}$ & $\#$ \\
\hline$x_{11}$ & 0 & $x_{43}$ & 3 & $x_{101}$ & 0 \\
$x_{12}$ & 0 & $x_{51}$ & $\mathbf{2}$ & $x_{102}$ & 3 \\
$x_{13}$ & $\mathbf{2}$ & $x_{52}$ & 0 & $x_{111}$ & 2 \\
$x_{21}$ & 3 & $x_{61}$ & 0 & $x_{121}$ & 5 \\
$x_{31}$ & 0 & $x_{62}$ & 1 & $x_{131}$ & 1 \\
$x_{32}$ & $\mathbf{1}$ & $x_{71}$ & $\mathbf{4}$ & $x_{132}$ & 1 \\
$x_{33}$ & $\mathbf{0}$ & $x_{81}$ & 7 & $x_{141}$ & 0 \\
$x_{34}$ & $\mathbf{3}$ & $x_{91}$ & 1 & $x_{142}$ & 0 \\
$x_{41}$ & 0 & $x_{92}$ & 0 & $x_{143}$ & 2 \\
$x_{42}$ & 0 & $x_{93}$ & 0 & $\mathbf{c x}=129, \mathbf{w x}=200$ \\
\hline
\end{tabular}

\subsubsection{Results comparisons}

For Examples 2 and 3, there are a total of four different solutions (expected reliability and max regret solutions for two different problems), and it is interesting to compare them. For all solutions without regard to the objective function, the maximum regret and expected system reliability were both computed. Furthermore the variance of system reliability, considering the uncertainty in future stress variables, was also computed. Table 14 presents the results from regret and expected reliability approaches for the two examples.

Table 14: Comparisons of solutions for Examples 2 and 3

\begin{tabular}{|l|c|c|c|c|}
\hline & \multicolumn{2}{|c|}{ Example 2 } & \multicolumn{2}{c|}{ Example 3 } \\
\hline & regret & expected reliability & regret & expected reliability \\
\hline objective function & 0.0274 & 0.8652 & 0.0257 & 0.9053 \\
\hline expected reliability & 0.8518 & 0.8652 & 0.8925 & 0.9053 \\
\hline max regret & 0.0274 & 0.0436 & 0.0257 & 0.0511 \\
\hline reliability variance & 0.032136 & 0.034099 & 0.0067438 & 0.0086379 \\
\hline cost & 122 & 130 & 122 & 129 \\
\hline weight & 200 & 200 & 200 & 200 \\
\hline
\end{tabular}

In the comparisons, the expected reliability is naturally higher when used as the objective function, and the maximum regret is naturally lower when it is the objective function. As expected, the riskneutral decision makers have higher expected reliability, while the risk-averse decision-makers have lower maximum regret. However, there are other very interesting results. Variance is a measure of 
uncertainty, and for decision making, uncertainty is aligned with risk. For Example 2, the variance is lower for the risk-averse case (minimize maximum regret) as would be expected, but they are very close. It could be argued based on the comparisons for Example 2, that both objective functions provide good solutions for both risk-averse and risk-neutral decision makers. However, the Example 3 results are different because the variance is almost 30\% higher for the expected reliability objective function, making it less desirable for risk-averse decision-makers.

\subsection{Experimental evaluation of the NS heuristic}

The problems can be solved using a global nonlinear solver or the NS heuristic or another effective optimization algorithm. If a global nonlinear integer solver is available, it should be used otherwise the NS heuristic is relative straightforward to implement and use. To evaluate the performance of the NS heuristic, we then solved additional variations for Example 2 with less than or equal to 14 subsystems to maximize expected system reliability. The performance of the NS heuristic was compared over a range of different problem sizes. Each problem was then also solved by B-and-B (nonlinear branchand-bound) and GA, to compare the performance of the NS heuristic. The optimization algorithms were run on different computers using different software, so computation time comparisons are difficult. For the problems with $s=1,2, \ldots, 5$, all four component choices were considered (the italicized numbers in Tables 2 to 4$)$.

Table 15 shows the expected system reliability values for the recommended solutions for six variations of the problem obtained using NS heuristic, B-and-B and GA. For $s=1$ and 2, global solver was also used. (LINGO Global Solver is also a branch-and-bound approach but designed to find the global, not local solution.) The performance of each method varies as we change the number of subsystems. Note that computation time for GA and NS increases significantly with problem size.

The comparisons are not conclusive and not generalizable. However, they indicate that the NS heuristic performs well compared to the other methods or certainty as well, and is relatively straightforward to implement. For the most difficult problem, the NS heuristic had higher objective 
function, but also with significantly more computation time than B-and-B.

Table 15: Expected system reliability obtained by using different search methods

\begin{tabular}{cc|cccc|cccc}
\hline \multicolumn{2}{c|}{ Example 2 } & \multicolumn{3}{c|}{ Expected System Reliability } & \multicolumn{4}{c}{ Computation Time (seconds) $^{1}$} \\
\hline$s$ & $C, W$ & NS & B-and-B & GA & global & NS & B-and-B & GA & global \\
\hline 1 & 5,10 & .9992 & .9992 & .9992 & .9992 & 0.529 & $<1$ & 0.594 & $<1$ \\
2 & 20,30 & .9885 & .9885 & .9885 & .9885 & 0.556 & $<1$ & 0.613 & $<1$ \\
3 & 40,60 & .9806 & .9806 & .9806 & -- & 0.626 & $<1$ & 1.457 & -- \\
4 & 60,80 & .9832 & .9832 & .9832 & -- & 0.698 & $<1$ & 2.791 & - \\
5 & 60,80 & .9757 & .9697 & .9757 & -- & 0.760 & $<1$ & 5.825 & - \\
14 & 150,200 & .8652 & .8631 & .7600 & -- & 28.62 & $<5$ & $1948.4^{2}$ & - \\
\hline
\end{tabular}

NOTES: (1) Personal computer with CPU Core i5-4210U $1.70 \mathrm{GHz}$

(2) GA with $s=14$ was also tested by developing custom code on a i7 Core personal computer. 12 runs were performed and GA was run for $>$ two hours. The best solution found was reliability $=0.8385, \operatorname{cost}=127$ and weight $=200$

\subsection{Discussion}

RAP was formulated considering uncertainty in future usage stresses. Two system reliability modeling approaches are implemented and the results obtained depending on the decision-maker risk preferences. Risk-averse designers or decision-makers can utilize the regret technique solutions. The decision-makers who desire good average system reliability results can adopt the risk-neutral formulation.

This paper presents system reliability optimization models which consider variations of component reliability caused by uncertain future stresses. In decision-making, the objectives are to minimize the maximum regret. Regret or opportunity loss is introduced to avoid consequences of a poor decision and the corresponding superior risk.

Acknowledgement - This study was based in part upon work supported by USA National Science Foundation (NSF) grants CMMI-0970140 and CMMI-0969423.

\section{References}

[1] D. Johnson, D. Coit, R. Kosaka \& K. Megow, "System Level Reliability Analyses and Predictions in a Varying Stress Environment, Proceedings of the 2013 Reliability \& Maintainability Symposium (RAMS), Orlando, FL, January 2013.

[2] A. Hada, System Reliability and Maintenance Modeling with Changing and Uncertain Future Stress Profiles, MS thesis, ISE, Rutgers University, 2012.

[3] A. Hada, D. Coit, M. Agnello \& K. Megow , "System Reliability Models with Stress Covariates for Changing Load Profiles,", Proceedings of the 2011 Reliability \& Maintainability Symposium (RAMS), Orlando, FL, January 2011.

[4] D. E. Fyffe, W. W. Hines, and N. K. Lee, "System reliability allocation problem and a computational algorithm," IEEE Trans. Rel., vol. 17, pp. 64-69, June 1968.

[5] P. M. Ghare and R. E. Taylor, "Optimal redundancy for reliability in series system”, Oper. Res., vol. 17, pp. 838-847, Sept. 1969. 
[6] M. Abouei Ardakan, A. Hamadani, Reliability optimization of series parallel systems with mixed redundancy strategy in subsystems, Reliability Engineering and System Safety, Elsevier 130 (2014) 132

[7] R. Soltani, S. J. Sadjadi, A. A. Tofigh, A model to enhance the reliability of the serial parallel systems with component mixing, Applied Mathematical Modeling, Elsevier 38 (2014) 1064-1076

[8] D. Coit, A. Smith, "Reliability optimization for series-parallel systems using a genetic algorithm", IEEE Trans. Rel., vol. 45, pp. 895-904, Sept. 1996.

[9] D. Coit, A. Smith, Considering Risk Profiles in Design Optimization for Series-Parallel Systems, Procs of the 1997 Reliabiliy \& Maintainability Symposium (RAMS), PA, Jan 1997

[10] D. Coit, A. Smith, "Redundancy Allocation to Maximize a Lower Percentile of the System Time-to-failure Distribution", IEEE Trans on Reliability, 47(1), 79-87, 1998.

[11] D. Coit, A. Smith, Genetic Algorithm to Maximize a Lower-Bound for System Time-to-Failure With Uncertain Component Weibull Parameters, Computers \& Ind Eng, vol. 41, no. 14, pp. 423-440, 2002

[12] D. Coit, Jin, T., Wattanapongsakorn, N., "System Optimization Considering Component Reliability Estimation Uncertainty: A Multi-Criteria Approach," IEEE Trans on Rel, 53(3), 369-380, 2004.

[13] M. Marseguerra, E. Zio, L. Podofillini,D. Coit., "Optimal design of reliable network systems in presence of uncertainty", IEEE Trans on Reliability 54(2): 243-253, 2005

[14] H. Tekiner, D. Coit, "System Reliability Optimization Considering Uncertainty: Minimization of a Coefficient of Variation Measure," Proceedings of the 2008 Industrial Engineering Research Conference, 995-1000, 2008.

[15] H. Tekiner, D. Coit, System Reliability Optimization Considering Uncertainty: Minimization of the Coefficient of Variation for Series-Parallel Systems. IEEE Trans on Rel 60(3), 2011.

[16] P. Wang, D. Coit, Reliability Assessment Based on Degradation Modeling with Random or Uncertain Failure Threshold, Proceedings 2007 Reliability \& Maintainability Symposium, Orlando, FL, Jan 2007.

[17] R. Rubinstein, G. Levitin, A. Lisniaski, and H. Ben-Haim, "Redundancy optimization of static series-parallel reliability models under uncertainty," IEEE Trans. Rel, vol. 46, no. 4, pp. 503-511, 1997.

[18] A. A. Taflanidis and J. L. Beck, "Stochastic subset optimization for reliability optimization and sensitivity analysis in system design," Computers and Structures, vol. 87, no. 5/6, pp. 318-331, 2009.

[19] A.K. Bhunia, L. Sahoo, D. Roy, Reliability stochastic optimization for a series system with interval component reliability via genetic algorithm, Applied Mathematics and Computation, Elsevier 216 (2010) 929-939.

[20] R. K. Gupta, A. K. Bhunia, and D. Roy, "A GA based penalty function technique for solving constrained redundancy allocation problem of series system with interval valued reliability of components," Journal of Computational and Applied Mathematics, vol. 232, no. 2, pp. 275-284, 2009.

[21] R. Soltani, S. Jafar Sadjadi, R. Tavakkoli-Moghaddam, Interval programming for the redundancy allocation with choices of redundancy strategy and component type under uncertainty: Erlang time to failure distribution, Applied Mathematics and Computation, 244:413-421, 01/2014

[22] M.J. Feizollahi, M. Modarres, The robust deviation redundancy allocation problem with interval component reliabilities, IEEE Transactions on Reliability, Vol 61 957-965, 2012

[23] L.J. Savage, The theory of statistical decision. Journal of American Statistical Association, 46(253), 55-67, 1951

[24] G. Loomes, R. Sugden, 'Regret theory: An alternative theory of rational choice under uncertainty', Economic Journal, 92(4), 805-24,1982

[25] D. Kahneman, A. Tversky, The psychology of preferences Scientific American, 246, 160-173, 1982

[26] D.E., Bell, Regret in Decision Making under Uncertainty. Operations Research 1982

[27] T. Gilovich, V.H. Medvec, The experience of regret: What, when, and why. Psychological Review, 102, 379-395, 1995

[28] T. Connolly, M. Zeelenberg, Regret in Decision Making, Department of Management and Policy, University of Arizona

[29] G. Chen, M. S. Daskin, Z. M. Shen, Stanislav Uryasev, The $\boldsymbol{\alpha}$-Reliable Mean-Excess Regret Model for Stochastic Facility Location Modeling, 28 July 2006 in Wiley InterScience 
[30] R. T. Moghaddam, M. K. Damghani, M. T Taghavifard, Decision Making under Uncertain and Risky Situations, Monographs, Apr. 2009

[31] M.J. Feizollahi, A. Shabbir, M. Mohammad, The Robust Redundancy Allocation Problem in Series-Parallel Systems With Budgeted Uncertainty, IEEE Transactions on Rel, vol 63 239-250, 2014

[32] N. Chatwattanasiri, D. Coit, M. Rodgers \& S. Song, System Reliability Optimization Considering Uncertain Future Operating Conditions and Usage Stresses, Proc of the 18th ISSAT International Conf on Reli and Quality in Design, Boston, MA, Jul 2012

[33] H. Markowitz, Portfolio selection. J. Finance 7, 77-91, 1952

[34] J. Dupacova, G. Consigli, S. Wallace, Scenarios for multistage stochastic programs. Ann. Oper. Res. 100 (2000) 25-53 (2001)

[35] B. Schroeder and G. Gibson. A large-scale study of failures in high-performance computing systems, Proc. of the 2006 International Conference on Dependable Systems and Networks (DSN'06), 2006.

[36] B. Schroeder and G. A. Gibson. Disk failures in the real world: What does an mttf of 1,000,000 hours mean to you?, Proceedings of 5th USENIX Conference on File \& Storage Technologies, February 2007.

[37] E. Pinheiro, W.D. Weber and L. Andr'e Barroso, Failure Trends in a Large Disk Drive Population, Proceedings of 5th USENIX Conference on File and Storage Technologies (FAST'07), February 2007

[38] P. Kouvelis, G. Yu, Robust Discrete Optimization and Its Applications, Kluwer Academic Publishers, Boston, 1997.

[39] C-V. Alfredo, A-M. Eduardo, M. Nelson, Minmax regret combinatorial optimization problems: an Algorithmic Perspective, RAIRO - Operations Research (2011) Volume: 45, Issue: 2, page 101-129.

[40] B. Craig, P. Relu, P.Pascal, S. Dale, Constraint-based Optimization with the Minimax Decision Criterion, CP, volume 2833 of Lecture Notes in Computer Science, page 168-182. Springer, 2003

[41] N. Chatwattanasiri, D. Coit, Q. Feng, Two-Stage Stochastic Model for System Reliability-Cost Optimization, Proceedings of the Ind \& Systems Engineering Research Conference, Canada, May 2014

[42] FEMTO-ST, IEEE PHM 2012 Data Challenge, http://www.femtost.fr/en/Research-departments/AS2M/Researchgroups/, PHM/IEEE-PHM-2012-Data-challenge.

[43] G. Levitin, L. Xing, Y. Dai, "Reliability and Mission Cost of 1-out-of- $N$ : $G$ Systems With State-Dependent Standby Mode Transfers", IEEE Trans. Rel., vol. 64, no. 1, pp. 895-904, March 2015.

[44] G. Levitin, L. Xing, Y. Dai, "Mission Cost and Reliability of 1-out-of- $N$ Warm Standby Systems With Imperfect Switching Mechanisms", IEEE Trans. Systems Man Cybernetics-Systems, vol. 44, no. 9, pp. 1262-1271, Sept. 2014.

[45] G. Levitin, L. Xing, Y. Dai, "Cold vs. hot standby mission operation cost minimization for 1-out-of- $N$ systems", European Journal of Operational Research, vol. 234, no. 1, pp. 155-162, April 2014.

[46] G. Levitin, L. Xing, Y. Dai, "Minimum Mission Cost Cold-Standby Sequencing in Non-Repairable Multi-Phase Systems", IEEE Trans. Rel., vol. 63, no. 1, pp. 251-258, March 2014. 